\title{
Electron beam modification of plasmonic responses of nanoparticles
}

\author{
Kevin Roccapriore ${ }^{1}$, Nicole Creange ${ }^{1}$, Shin Hum $\mathrm{Cho}^{2}$, Delia Milliron ${ }^{3}$ and Sergei Kalinin ${ }^{4}$
}

${ }^{1}$ Oak Ridge National Laboratory, United States, ${ }^{2}$ Samsung Electronics, United States, ${ }^{3}$ University of Texas at Austin, United States, ${ }^{4}$ Center for Nanophase Materials Sciences, Oak Ridge National Laboratory, Oak Ridge, Tennessee, United States

Nanoparticles can exhibit exotic physics, display quantum phenomena, and offer opportunities in multiple disciplines and applications. Plasmonics is one field in which nanoparticles has made a large impact, for example in biosensing, the pharmaceutical industry, and quantum optics. Particles are typically chemically synthesized and can be tailored to meet specific plasmon resonance requirements based on the chemical recipe. Resonances of nanoparticles can extend from the near infrared (NIR) to the ultraviolet (UV) spectral regions and can even be spectrally tunable based on the level of incorporated dopants [1]. However, once synthesized, the plasmon response cannot be dynamically modified. Here we report use of the electron beam in a scanning transmission electron microscope (STEM) to modify plasmon resonances of doped semiconductor nanocrystals in both space and energy.

Using the framework of a self-assembled system of nanoparticles, we direct the electron beam at higher than normal operating currents in order to intentionally modify individual nanoparticles. Previous work related to the use of an electron beam for modification of structures has shown precise cutting and welding of metallic and silicon nanowires [2], as well as positioning of atoms and dopants within two dimensional materials [3], however, here we focus on the application to nanoparticle plasmonics, and attempt to understand the mechanisms responsible for modification, e.g., chemical changes. As an example, in Figure 1, we demonstrate intentional removal of specific nanoparticles within a self-assembled nanoparticle array in order to reveal various designed structures. In this case, individual particles are completely removed, as seen in the high angle annular dark field (HAADF) images in Fig 1 (a,e,i), while simultaneously monochromated electron energy loss (EEL) spectrum images are acquired. We use non-negative matrix factorization (NMF) to quickly display the dominant spectral features in the data, and the resulting energy maps after exposure are depicted below the corresponding HAADFs. Note that entire particles need not be removed, and instead small portions of individual particles can be selectively removed.

In addition to modifying plasmon response in the spatial domain, we also show it is possible to dynamically tune the spectral response using the electron beam. In Figure 2, a pair of nanoparticles is irradiated with a defocused electron beam positioned at the junction between the two, and HAADFs and STEM-EELS maps are collected over discrete time intervals. Again, we use NMF to spectrally deconvolve the EELS signal, and find that not only do the particles begin to fuse, but also all dominant NMF modes begin to exhibit a redshift. After performing core-loss EELS experiments during irradiation, we postulate that loss of metallic atoms in the structure reduces the effective electron density which has the effect of reducing the plasma frequency.

Finally, in order to elicit a more controllable platform and hence gain more control over nanoparticle plasmonics, we have developed a flexible automated experiment based in a Python environment where individual particles can be selected from a HAADF image, and subsequently removed automatically - partially or completely. This method incorporates different methods of feedback to ensure appropriate particle removal rates, thus further enabling very specific dynamic tuning of these plasmon resonances. The ability to adjust the particle response on the fly significantly increases the design possibilities for nanostructure development and is key for nanophotonic design. 
a)

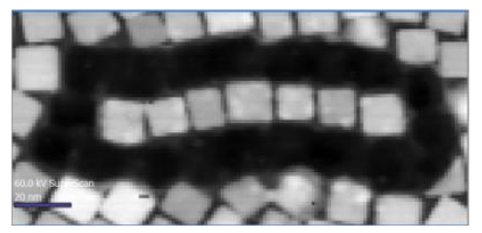

b)

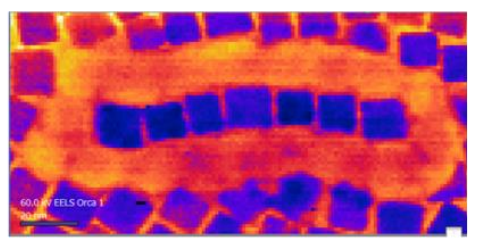

c)

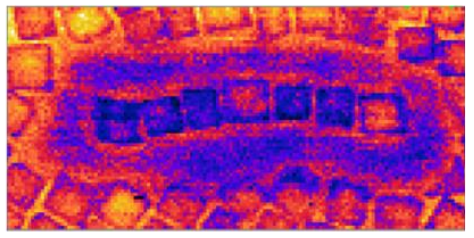

d)

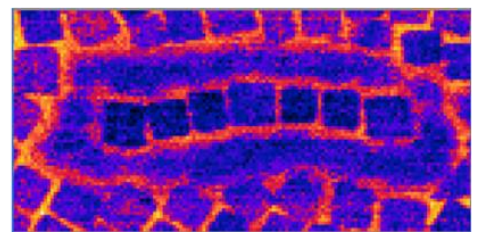

e)

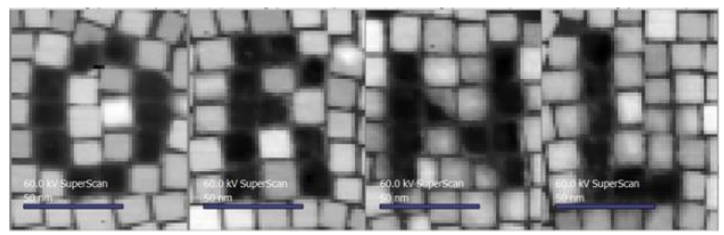

f)

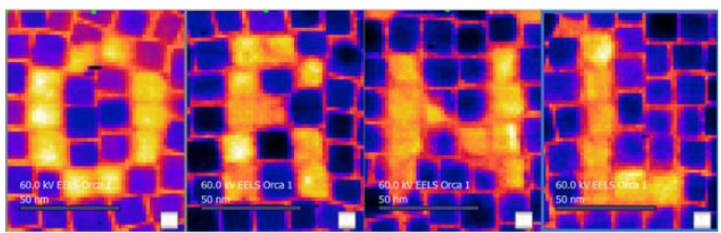

g)

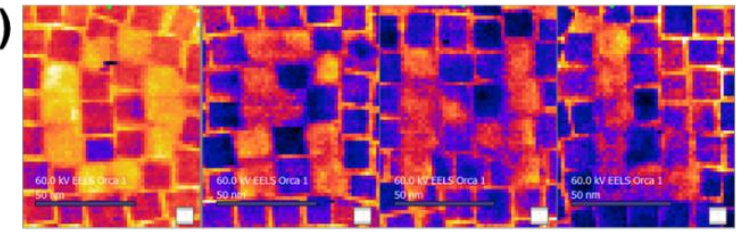

h)

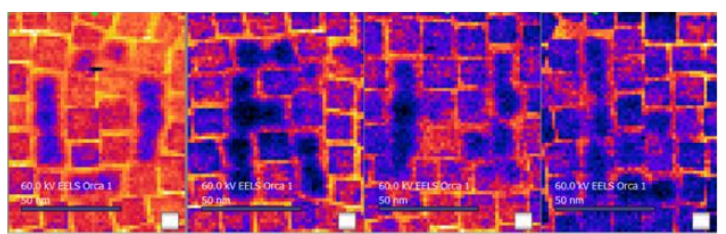

i)

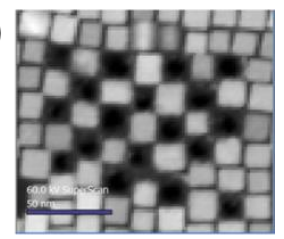

j)

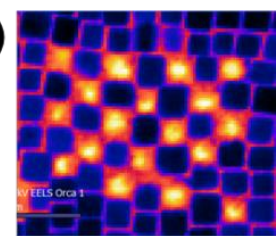

k)

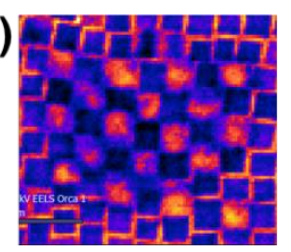

I)

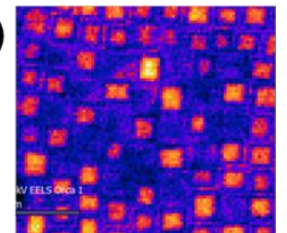

Figure 1. Figure 1. Examples of electron beam modification to plasmon resonances in space. (a, e, and j) are high angle annular dark field (HAADF) images acquired after irradiating targeted nanoparticles. (b,f,j), (c,g,k), and $(\mathrm{d}, \mathrm{h}, \mathrm{i})$ represent the dominant three NMF components arising after electron beam exposure.

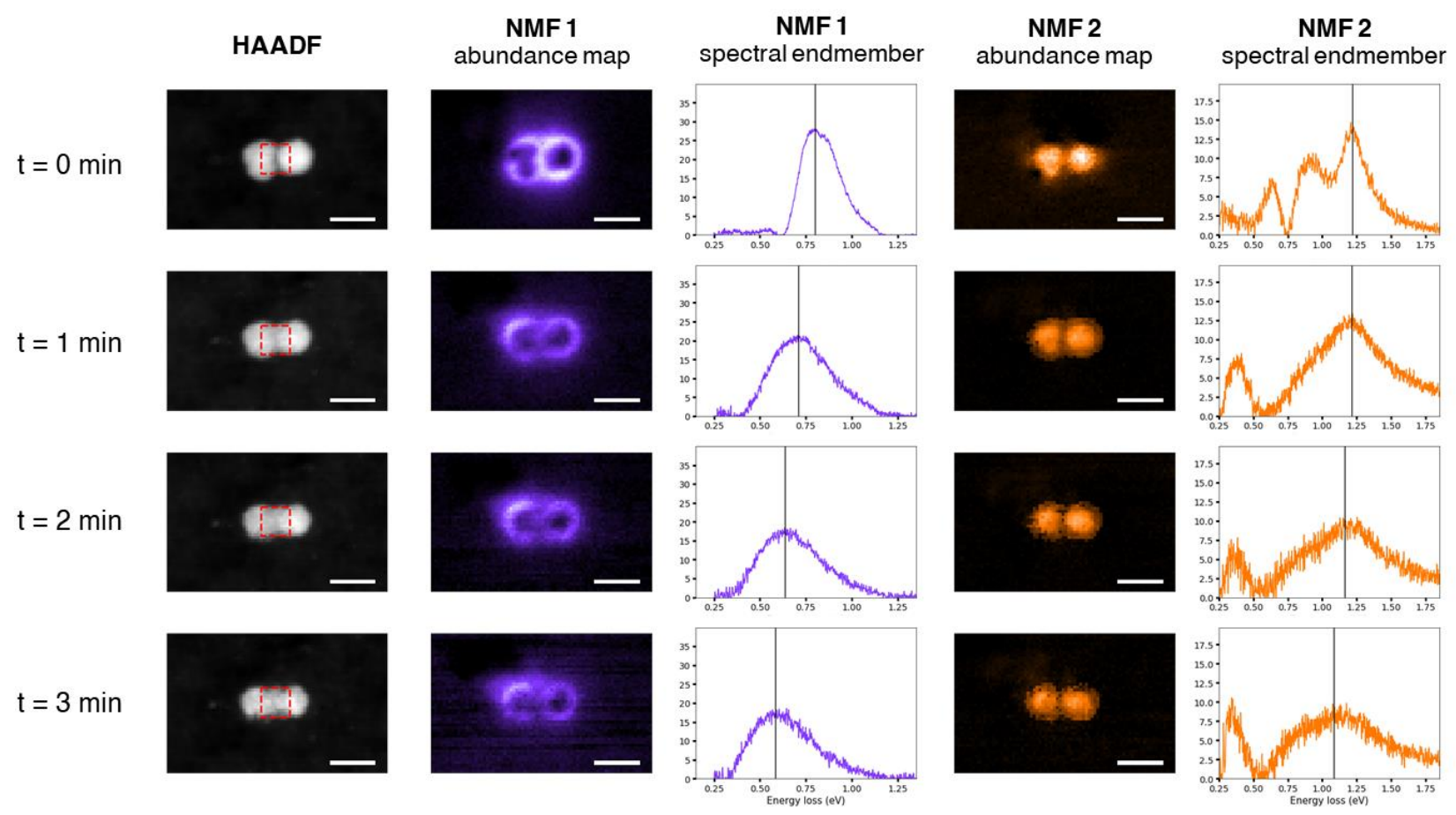


Figure 2. Figure 2. Evolution of a nanoparticle dimer pair upon defocused electron beam irradiation, where dimer fuses together over sufficient exposure. Plasmon resonances revealed by non-negative matrix factorization (NMF) show both dominant plasmon modes are modified in the spectral domain (red-shifted).

References

[1] S. H. Cho et al., J., Chem. Mater.201931, 2661.

[2] S. Xu et al. Small20051, 1221.

[3] O. Dyck et al. Nat. Rev. Mat. 20194, 497.

[4] This effort (ML and STEM) is based upon work supported by the U.S. Department of Energy (DOE), Office of Science, Basic Energy Sciences (BES), Materials Sciences and Engineering Division (K.M.R., S.V.K.) and was performed and partially supported (J.A.H., M.Z.) at the Oak Ridge National Laboratory's Center for Nanophase Materials Sciences (CNMS), a U.S. Department of Energy, Office of Science User Facility. S.H.C acknowledges (NSF, CHE-19052631609656, CBET-1704634, NASCENT, an NSF ERC EEC1160494, and CDCM, an NSF MRSEC DMR-1720595), the Welch Foundation (F-1848), and the Fulbright Program (IIE-15151071). 